\title{
From Solid-State NMR to Crystal Structures through Combinatorial Tiling Theory
}

Darren Brouwer*, Janelle Vanderhout, Chelsey Hurst, Brydon Eastman

Department of Chemistry, Redeemer University College

Solid-state NMR spectroscopy can provide a great deal of structural information about the local environments of NMR-active nuclei. When combined with other complementary techniques such as diffraction and quantum chemical calculations - an approach referred to as NMR crystallography powerful structure determination strategies emerge that allow for the elucidation of detailed structures that no one technique could provide on its own. We have been particularly interested in developing structure solution strategies that maximally exploit the structural information available in $1 D$ and $2 D$ solid state NMR data from network materials such as zeolites, with a view to being able to solve structures when minimal diffraction data is available, such as disordered layered structures. Based on some elegant mathematics in the area of combinatorial tiling theory, we are developing a novel structure solution strategy for network materials that will efficiently generate all feasible structures that arise from the number of sites (number of peaks), relative site occupancies (peak intensities), coordination environments (chemical shifts) revealed in 1D solid-state NMR spectra and the inter-site connectivities (cross peak intensities) revealed in 2D correlation spectra. One of the key features of this approach is that no prior knowledge of the crystallographic space group or unit cell parameters is intrinsically necessary, which in principle allows for the generation of network crystal structures directly from solid-state NMR data alone. This talk will introduce and explain Delaney symbols (mathematical structures for describing tilings of the 2D plane or 3D space), highlighting the rich amount of structural information that they contain and how they allow for novel conceptual linkages between solid-state NMR and crystallography of network materials.

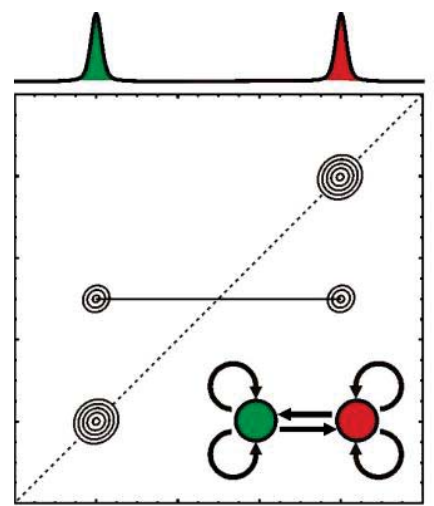

Solid-state NMR

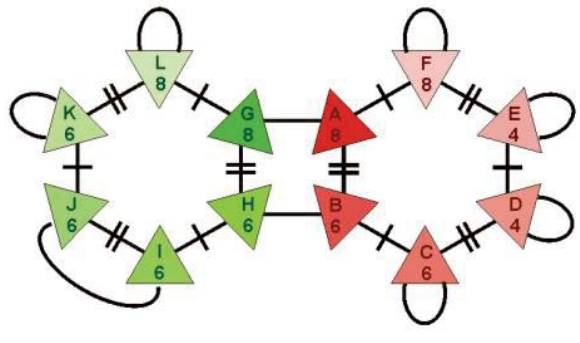

Tiling Theory

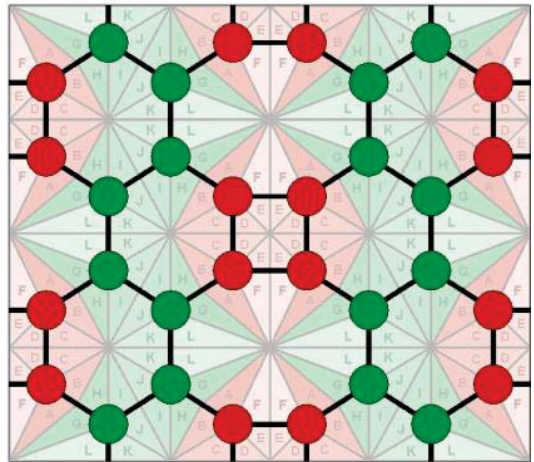

Structure 